\title{
Human Skill Quantification for Excavator Operation using Random Forest
}

\author{
Hiromu Imaji \\ Graduate School of Engineering, Hiroshima University, \\ 1-4-1, Kagamiyama, Higashihiroshima City, Hiroshima, 739-8527, Japan \\ E-mail:imaji-hiromu@hiroshima-u.ac.jp \\ http://www.hiroshima-u.ac.jp \\ Kazushige Koiwai, Toru Yamamoto \\ Academy of Science and Technology, Hiroshima University, \\ 1-4-1, Kagamiyama, Higashihiroshima City, Hiroshima, 739-8527, Japan \\ E-mail:koiwaik@hiroshima-u.ac.jp,yama@hiroshima-u.ac.jp \\ http://www.hiroshima-u.ac.jp \\ Koji Ueda, Yoichiro Yamazaki \\ Global Engineering Center, KOBELCO Construction Machinery CO., LTD., \\ 2-2-1, Itsukaichikou, Saeki-ku, Hiroshima City, Hiroshima, 731-5161, Japan
}

\begin{abstract}
In the construction field, the improvement of the work efficiency is one of important problems. However, the work efficiency using construction equipment depends on their operation skills. Thus, in order to increase the work efficiency, the operation skill is required to be quantitatively evaluated. In this study, the Random Forest, one of machine learning method, is adopted as the quantitatively evaluation for the operation skill of construction equipment. Evaluated target is the operation on an excavation to load onto a truck for a hydraulic excavator. The Random Forest learns to classify some states by the pilot pressure of skilled worker's operation. States are defined as 'dig', 'lift', 'dump', 'reposition', and 'idle'. The Random Forest with the learning result of skilled worker is applied to other worker's operation. The human skill quantification is verified based on the 'idle' state.
\end{abstract}

Keywords: human skill, machine learning, random forest, hydraulic excavator

\section{Introduction}

In the field of construction industry, the application of Information and Communication Technology (ICT) for a construction equipment has been proceeded to improve the productivity, which is called 'i-Construction' in Japan. ${ }^{1}$ Automation technologies are important to improve the work efficiency. ${ }^{2}$ However, work efficiencies of some construction equipment still depend on operation skills for each worker. Therefore, it is necessary to establish a method to evaluate the operation skills so that it is possible to improve the productivity in the field of construction industry.

In previous studies, the human skill evaluation based on the control engineering approch ${ }^{3}$ has been proposed and the evaluation of operation skills based on interviews has been investigated. ${ }^{4}$ However, these methods focus on the qualitative evaluation only and it is difficult to utilize them to improve the productivity.

In this paper, the quantification method of operation skills for construction machines based on classifications of operation state using the Random Forest (RF) is 
proposed. The proposed method is applied to the excavator operation.

$\mathrm{RF}$ is a kind of machine learning methods proposed by Leo Breiman in 2001. ${ }^{5} \mathrm{RF}$ is constructed by some decision trees. Since the decision tree is an aggregate of branching rules, it is widely used to perform data mining for the certain classification problems because the learning result is more readable than other machine learning methods. ${ }^{6,7}$ Moreover, RF has the lower computational cost for the classification.

\section{Proposed method}

\subsection{Classification of decision tree}

The learning data for the decision tree is comprised of a set $X$ and a set $Y$. The set $X$ consists of feature vectors, $\boldsymbol{x}_{i}$, which are one-dimensional vectors included the feature of data. The set $Y$ consists of natural number, $y_{i}$, which are labels for the classification. Those formula are shown by:

$$
\begin{gathered}
X=\left\{\boldsymbol{x}_{i} \mid i=1,2, \ldots, n\right\}, \\
\boldsymbol{x}_{i}=\left\{x_{i j} \mid j=1,2, \ldots, m\right\} \\
Y=\left\{y_{i} \mid i=1,2,3, \ldots, n\right\}, \\
y_{i}=1,2,3, \ldots, K
\end{gathered}
$$

where $n$ is defined as the number of feature vectors, $m$ is defined as the dimensions of the feature vectors and $K$ is defined as the number of labels. A set of training data, $T$ is defined as follows:

$$
\begin{gathered}
T=\left\{t_{i} \mid i=1,2,3, \ldots, N\right\}, \\
t_{i}=\left\{\boldsymbol{x}_{i}, y_{i}\right\}
\end{gathered}
$$

here, $\mathrm{N}$ is donated as the number of training data. $\mathrm{N}$ must be smaller than $\mathrm{n}$.

\subsubsection{Training procedure}

The set of initial training data is shown as $T_{0}$. The branch part is expressed as a node $N_{l}$, and the initial node is $N_{0}$. The learning procedure is shown for each step below.

[Step 1-1] Sampling feature vector

The sampled feature vector given as follow:

$$
\widehat{\boldsymbol{x}}_{i}=\left\{x_{i j} \mid j \in J\right\}
$$

where $J$ is a set of $\widehat{m}$, whose components are natural numbers selected randomly without overlap among $1,2,3, \ldots, m$.

[Step 1-2] Determination of branching condition

$\bar{J}$ is defined as the selected index of feature vector element. $c$ is defined as the threshold that divide the training data into two. The selected index, $\bar{J}$, and the threshold, $c$, have the following conditions:

$$
\bar{J} \in J
$$

$$
\min \left(x_{i \bar{\jmath}}\right)<c \leq \max \left(x_{i \bar{\jmath}}\right)
$$

$T$ is divided into a set $T_{1}$ with $x_{i \bar{\jmath}} \leq c$ and a set $T_{2}$ with $x_{i \bar{j}}>c$.Then, $\bar{j}$ and $c$ which minimize the following $\Phi\left(T, T_{i}\right)$ are obtained.

$$
\begin{gathered}
\Phi\left(T, T_{i}\right)=I(T)-\sum_{k=1}^{2} P\left(T_{k} \mid T\right) I\left(T_{k}\right) \\
I(T)=1-\sum_{k=1}^{\mathrm{K}} P^{2}\left(C_{k} \mid T\right)
\end{gathered}
$$

here $P(A \mid B)$ is the ratio of elements $A$ in $B . C_{k}$ is a set of $t_{i}$ with label $k$. $\hat{\jmath}$, and $\hat{c}$ are obtained with the state of minimized $\Phi\left(T, T_{i}\right)$.

[Step 1-3] Save training data

$\hat{\jmath}, \hat{c}$, and $T$ are saved at each node of $N_{l}$.

[Step 1-4] End judgment

When $T_{1}$ or $T_{2}$ do not satisfy the following termination condition shown below, Step 1-1 is repeated with $T_{1,2} \rightarrow T$.

Termination condition:

(i) $T_{1}$ or $T_{2}$ has a only single label.

(ii) The number of data in the data set is less than or equal to a certain value.

(iii) The certain depth is achieved at a branch.

\subsubsection{Classification procedure}

To classify the data, the feature vector of validation is defined as $\overline{\boldsymbol{x}}=\left\{\bar{x}_{j} \mid j=1,2, \ldots, m\right\}$.

[Step 2-1] Input the feature vector to RF $\overline{\boldsymbol{x}}$ input to the first node of the trained RF. 


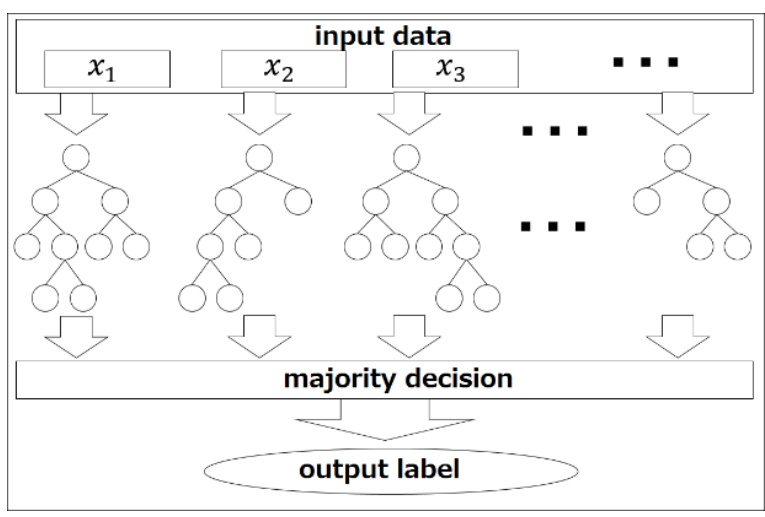

Fig. 1 Scheme of Random Forest

[Step 2-2] Select the node

Based on the value of $\overline{\boldsymbol{x}}, \hat{\boldsymbol{j}}$ and $\hat{\boldsymbol{c}}$, the node is selected until the end of node.

[Step 2-3] Output of results

The largest number of label $\hat{y}$ in $T$ at the end of node should be the result of classification of the decision tree.

\subsection{Random Forest}

The scheme of RF is shown in Fig. 1. The detail of RF is explained below.

\subsubsection{Training of Random Forest}

$\mathrm{RF}$ is composed of a plurality of decision trees. Therefore, RF needs to generate training data of decision trees. It is assumed that training data is given to $\mathrm{RF}$ as eq. (1) and (2). Thus, a training data of the decision tree in $\mathrm{RF}$ is given as follows:

$$
\begin{gathered}
\hat{X}=\left\{\boldsymbol{x}_{i} \mid i \in I\right\}, \\
\hat{Y}=\left\{\hat{y}_{i} \mid i \in I\right\}
\end{gathered}
$$

where $I$ is a set of $\hat{n}$, whose components are natural numbers selected randomly without overlap among $1,2,3, \ldots, n$. The data sets expressed by eq.(9) is generated for the number of decision trees and used for training each decision tree. The overlapping between data of decision trees is allowed.

\subsubsection{Quantification}

$N$ is defined as the total number of decision trees. $L$ is defined as the number of decision trees outputting the specific label. The ratio of $L$ in $N$ is shown as $Q$, below:

$$
Q=L / N
$$

The result of ratio can express the quantification.

\section{Experiment Evaluation}

\subsection{Experimental device}

The experiment focused on the quantification of the operation skill by the hydraulic excavator. As shown in Fig. 2, it is known that the general work of loading dump of earth and sand with a hydraulic shovel is classified into four states of "dig", "lift", "dump" and "reposition". This study classifies states of the work into 5 states, which are above states with "idle". The "idle" state shows no operation.

The operation of the hydraulic excavator is composed of four operations of "bucket", "arm", "boom", and

\section{Dig}

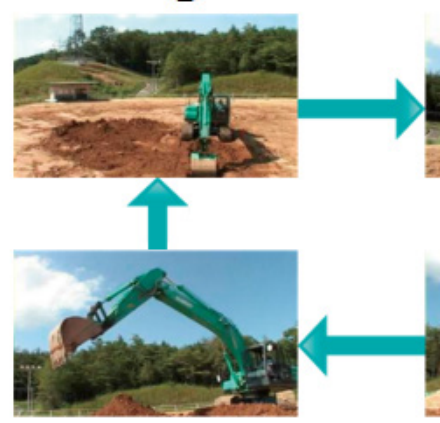

Reposition
Lift

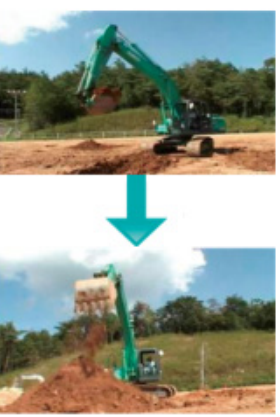

Dump
Fig. 2 General hydraulic excavator excavation work

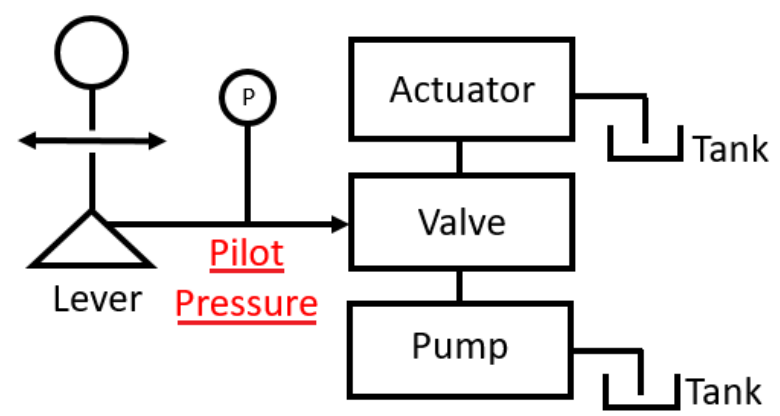

Fig. 3 Typical hydraulic system on a excavator 
"swing". These input signals of operations were acquired as pilot pressures. The pilot pressure is the input pressure to the valve corresponding to the lever operation amount. A simple configuration diagram of the hydraulic system is shown in Fig. 3. In this paper, eight types of pilot pressures, the bucket dig, the bucket dump, the boom up, the boom down, the arm pull, the arm push, the right swing and the left swing are adopted as elements of the feature vector to be used for RF.

\subsection{Experimental procedure and result}

The experimental procedure in this paper is shown below.

\section{[Step 1]}

Subjects, which are the skilled worker, the general worker and the unskilled worker, operate the excavator to perform the excavation and the mimic loading onto a dump truck shown in Fig. 2 with several cycles. Their data of pilot pressure are obtained.

\section{[Step 2]}

Training data of pilot pressure as feature vectors are extracted from the data of skilled worker. The extracted training data is one cycle only. Labels of 5 states, which are "dig", "lift", "dump", "reposition" and "idle", for the training to RF is set by the manual processing of data to check the change of pilot pressure. Those training data
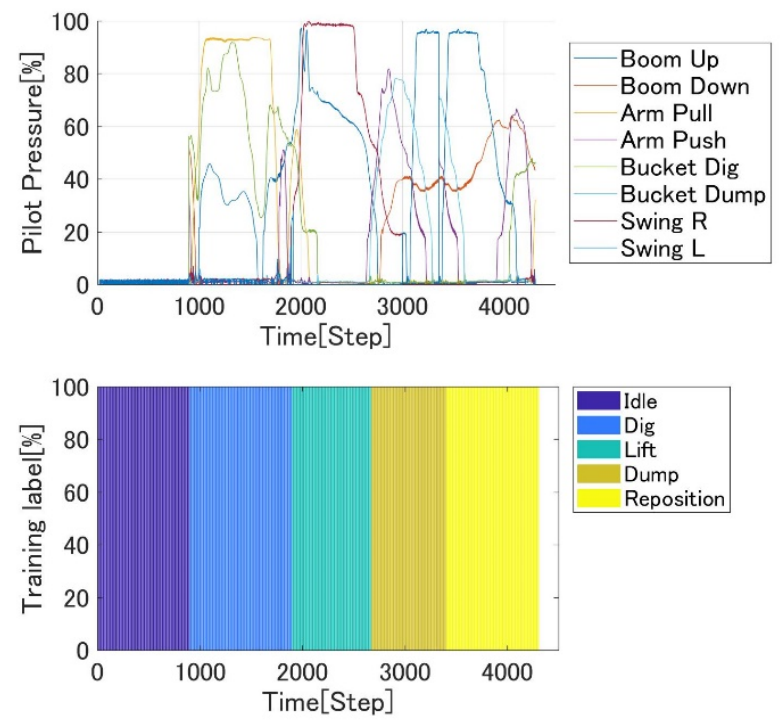

Fig. 4 Training data for RF are shown in Fig. 4. Here, values of pilot pressure are normalized at the $100 \%$ of the maximum value. By using feature vectors and labels, RF learns labels of 5 states by pilot pressures of a skilled worker. Table 1 shows parameters of the proposed method.

\section{[Step 3]}

Each operation data of the unskilled worker, the general worker, and the skilled worker are extracted to classified by RF. The extracted data of the skilled worker does not include the training data. Moreover, extracted data are almost one cycle of Step 1. Using the Result of Step 2, each extracted data of the unskilled worker, the general worker, and the skilled worker are classified. At this time, the ratio of the predicted label is obtained. Those extracted data and results of classification are shown in Fig. 5, Fig. 6 and Fig. 7 respectively.

Table 1 parameters of experiment

\begin{tabular}{|l|c|}
\hline Step of training data & 4309 \\
\hline Step of verification data & 4000 \\
\hline Number of decision trees & 31 \\
\hline $\begin{array}{l}\text { Number of sampling data for } \\
\text { one decision tree }\end{array}$ & 1436 \\
\hline $\begin{array}{l}\text { Number of sampling feature } \\
\text { for one decision tree }\end{array}$ & 3 \\
\hline
\end{tabular}
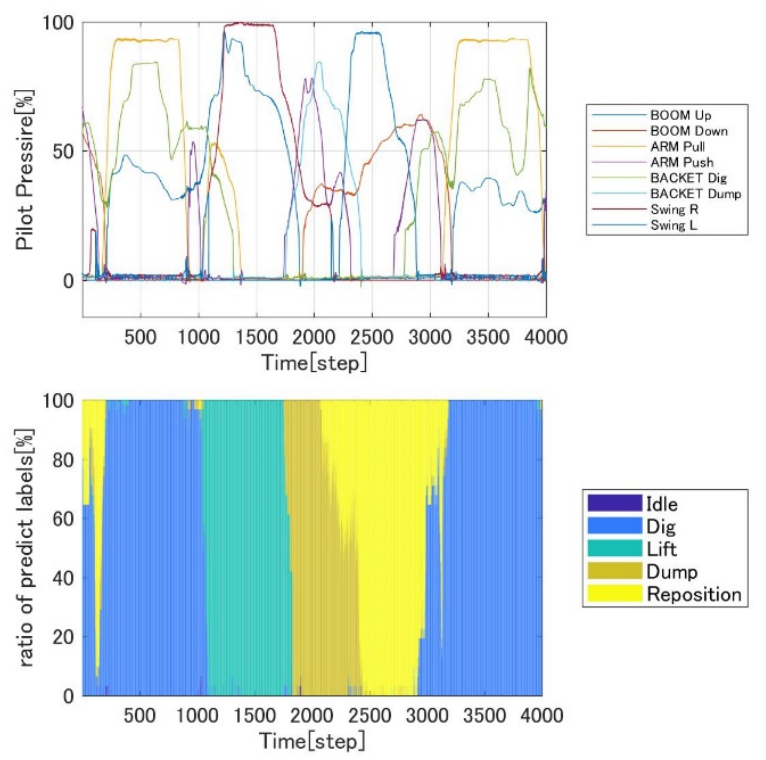

Fig. 5 Learning result of skilled worker using RF 

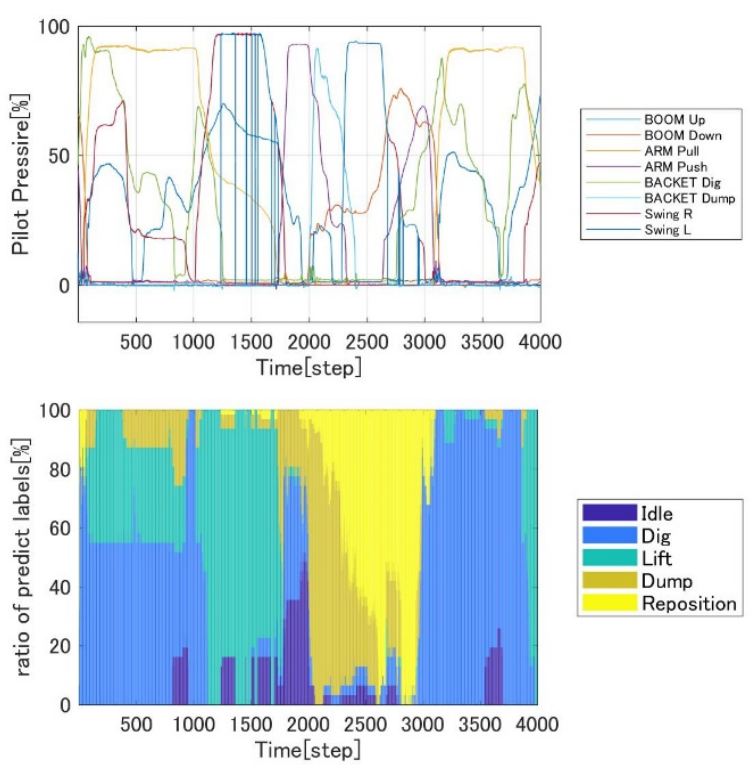

Fig. 6 Learning result of general worker using RF
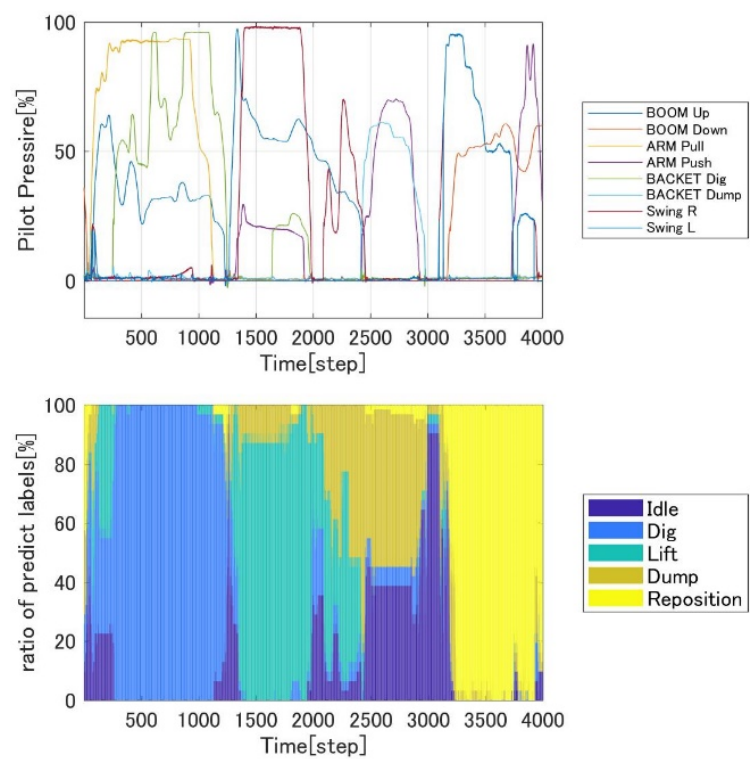

Fig. 7 Learning result of unskilled worker using RF

\subsection{Skill quantification}

Predict labels in Fig. 5, Fig. 6, and Fig. 7 are considered as the skill quantification. The skilled worker data is clear to classify each states without the "idle" state. However, predict labels of the general
Table 2 Ratio of idle

\begin{tabular}{|c|c|c|c|}
\hline worker & Skilled & General & Unskilled \\
\hline \hline $\begin{array}{c}\text { Area Ratio } \\
{[\%]}\end{array}$ & 0.09 & 5.32 & 13.7 \\
\hline
\end{tabular}

Table 3 Verification result of RF significance

\begin{tabular}{|l|c|c|c|}
\hline worker & Skilled & General & Unskilled \\
\hline \hline average & 0.15 & 3.45 & 13.3 \\
\hline Variance & $\begin{array}{l}2.92 \\
\times 10^{-5}\end{array}$ & $\begin{array}{l}1.48 \\
\times 10^{-2}\end{array}$ & $\begin{array}{l}2.06 \\
\times 10^{-2}\end{array}$ \\
\hline Max & 0.26 & 6.74 & 15.9 \\
\hline Min & $\begin{array}{l}5.00 \\
\times 10^{-2}\end{array}$ & 1.70 & 10.5 \\
& \multicolumn{2}{|l}{} \\
\hline
\end{tabular}

worker and the unskilled worker have some of the "idle" state in spite of working. Therefore, the state "idle" is quantified. Table 2 shows the result of quantification, that is the ratio of decision trees with the "idle" state. Moreover, Table 3 shows the result of training with 50 times using the same input / output data. This result shows the validity of RF. The ratio of decision trees with "idle" is getting bigger from the skilled worker to the unskilled worker in Table 2 and Table 3.

The dump operation that have the larger difference of the "idle" state, therefore around dump motions are extracted in Fig. 8, Fig. 9 and Fig. 10 respectively. In Fig. 9, the "idle" state appears around 1900 step of the general worker. To see the pilot pressure, the operation of the general worker is only single operation of the arm push at the appearing "idle" state. The skilled worker has the almost three operations at the same period. In Fig. 10, the unskilled worker performs one or two operations only. Moreover, no operation is there around 3000 steps when a state changes form the dump to the reposition. Those less operation compared with the skilled operator is expressed by the amount of the "idle" state. Thus, the ratio of "idle" state represents the lack of operation in the work.

\section{Conclusion}

The human skill quantification for the excavator operation using the random forest has been proposed. The random forest learns the data of the skilled operator as the feature vector and labels of work state. Based on 


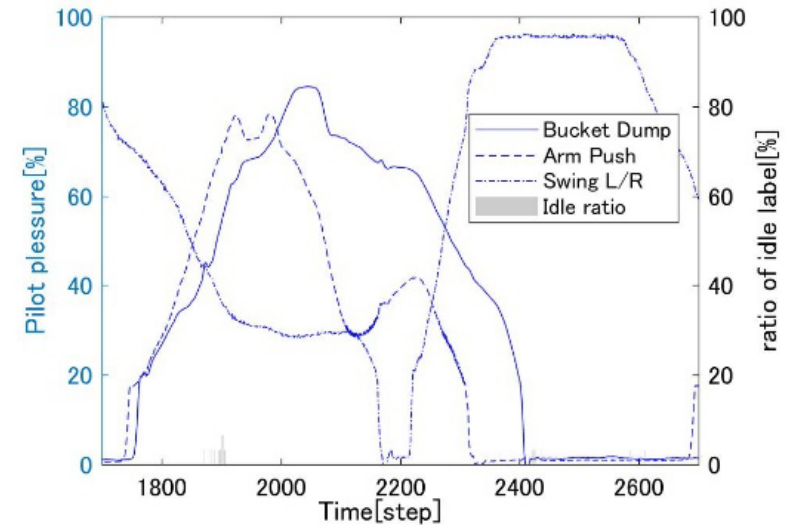

Fig. 8 Part of pilot pressure by skilled worker

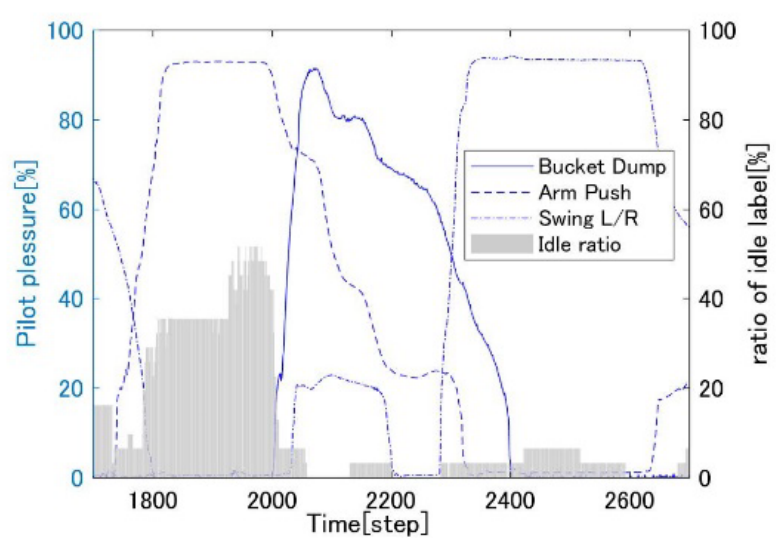

Fig. 9 Part of pilot pressure by general worker

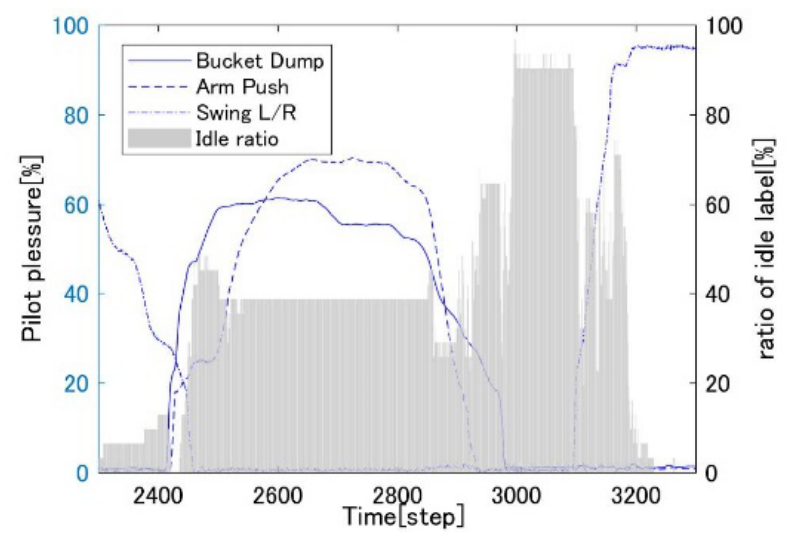

Fig. 10 Part of pilot pressure by unskilled worker the learned random forest, work statements are classified by using data of each worker, which are the skilled operator, the general operator, and the unskilled operator. Results of classifications have been considered and it is clear that the amount of the "idle" state is different. The "idle" state seem to express the lack of operation compared with the skilled operator. As the future work, other states are classified by using internal state and output. Moreover, the relation of any model such as the Hidden Markov Model will be researched. Furthermore, the method can be utilized to improve the work efficiency.

\section{References}

1. T. Kakizaki, i-Construction: On productivity improvement of construction site (in Japanese), Monthly construction, 60(1) (2016) 6-9.

2. Y.Sakaida, D.Chugo, H.Yamamot, H.Asama: The Analysis of Excavator Operation by Skillful Operator; Proc. of SICE Annual Conference, Tokyo, (2008) 538-542

3. K. Koiwai, Y. Liao, T. Yamamoto, T. Nanjo, Y. Yamazaki, and Y. Fujimoto, Feature extraction for excavator operation skill using CMAC, Journal of Robotics and Mechatronics, 28(5) (2016) 715-721.

4. Y. Kawazoe, K. Enomoto, J. Matsumoto, S. Okabe, and S. Handa, Acquisition of Human Operator's Skills and Dexterity using Neural Network: Automatic Generation of Neural Controller from Chaotic Time Series Data during Stabilizing Control of an Inverted Pendulum, Proc. of 29th SICE Symposium on Intelligent Systems, (2002) 217-222.

5. L Breiman, Random Forests, Machine Learning, 45(1) (2001) 5-32

6. J. Kim, J. Lee, and Y. Lee, Data-mining-based coronary heart disease risk prediction model using fuzzy logic and decision tree, Healthcare Informatics Research, 21(3) (2015) 167-174.

7. X. Wang, X. Liu, W. Pedryc, and L. Zhang, Fuzzy rule based decision trees, Pattern Recognition, 48(1) (2015) 50-59. 